\title{
Accuracy, Precision, and Trending Ability of Electrical Cardiometry Cardiac Index versus Continuous Pulmonary Artery Thermodilution Method: A Prospective, Observational Study
}

\author{
P. B. W. Cox, ${ }^{1}$ A. M. den Ouden, ${ }^{2}$ M. Theunissen, ${ }^{1}$ L. J. Montenij, ${ }^{3}$ A. G. H. Kessels, ${ }^{1}$ \\ M. D. Lancé, ${ }^{4}$ W. F. F. A. Buhre, ${ }^{1}$ and M. A. E. Marcus ${ }^{4}$ \\ ${ }^{1}$ Department of Anesthesiology and Pain Management, Maastricht University Medical Center+, \\ Prof. Debyelaan 25, 6202 AZ Maastricht, Netherlands \\ ${ }^{2}$ Department of Anesthesiology and ICU, St. Antonius Ziekenhuis, Postbus 2500, 3430 EM Nieuwegein, Netherlands \\ ${ }^{3}$ Department of Anesthesiology, University Medical Centre Utrecht, Heidelberglaan 100, 3584 CX Utrecht, Netherlands \\ ${ }^{4}$ Department of Anesthesiology, ICU and Perioperative Medicine, HMC, P.O. Box 3050, Doha, Qatar
}

Correspondence should be addressed to P. B. W. Cox; b.cox@mumc.nl

Received 28 April 2017; Revised 21 July 2017; Accepted 20 August 2017; Published 9 October 2017

Academic Editor: Kazunori Uemura

Copyright (C) 2017 P. B. W. Cox et al. This is an open access article distributed under the Creative Commons Attribution License, which permits unrestricted use, distribution, and reproduction in any medium, provided the original work is properly cited.

\begin{abstract}
Introduction. Evaluation of accuracy, precision, and trending ability of cardiac index (CI) measurements using the Aesculon ${ }^{\mathrm{TM}}$ bioimpedance electrical cardiometry (Aesc) compared to the continuous pulmonary artery thermodilution catheter (PAC) technique before, during, and after cardiac surgery. Methods. A prospective observational study with fifty patients with ASA 3-4. At six time points $(\mathrm{T})$, measurements of CI simultaneously by continuous cardiac output pulmonary thermodilution and thoracic bioimpedance and standard hemodynamics were performed. Analysis was performed using Bland-Altman, four-quadrant plot, and polar plot methodology. Results. CI obtained with pulmonary artery thermodilution and thoracic bioimpedance ranged from 1.00 to $6.75 \mathrm{~L} \mathrm{~min}^{-1}$ and 0.93 to $7.25 \mathrm{~L} \mathrm{~min}^{-1}$, respectively. Bland-Altman analysis showed a bias between $\mathrm{CI}_{\mathrm{BIO}}$ and $\mathrm{CI}_{\mathrm{PAC}}$ of 0.52 liters $\min ^{-1} \mathrm{~m}^{-2}$, with LOA of $[-2.2 ; 1.1]$ liters $\mathrm{min}^{-1} \mathrm{~m}^{-2}$. Percentage error between the two techniques was above $30 \%$ at every time point. Polar plot methodology and 4-quadrant analysis showed poor trending ability. Skin incision had no effect on the results. Conclusion. CI obtained by continuous PAC and CI obtained by Aesculon bioimpedance are not interchangeable in cardiac surgical patients. No effects of skin incision were found. International clinical trial registration number is ISRCTN26732484.
\end{abstract}

\section{Introduction}

Measurement of cardiac output (CO) and cardiac index (CI) is commonly used in patients undergoing cardiac surgery. The continuous pulmonary artery thermodilution technique is well known as a method for advanced monitoring of cardiovascular function and is regularly used as a clinical reference technique in method comparison studies. However, a couple of studies during the past years showed that a true golden standard for measuring cardiac output does not exist.

Due to its invasive nature, the use of the PAC is associated with severe risk and there is still discussion on whether the positive effects of PAC outweigh the adverse effects [1-5].
Today, there are less invasive or even noninvasive monitoring devices available. The ideal technique should be reliable, noninvasive, continuous, cost-effective, and user independent and should have a fast response time enabling rapid detection of hemodynamic changes [6]. Considering the growing age of the surgical population with severe comorbidity, it is likely that monitoring of $\mathrm{CO}$ will be important, also in non-cardiac-surgery patients. Moreover, assessment of $\mathrm{CO}$ and stroke volume (SV) is a prerequisite to establish early goal-directed therapy during the perioperative period.

One of the most recent noninvasive techniques for assessment of $\mathrm{CO}$ is based on a modified thoracic bioimpedance algorithm. Briefly, thoracic bioimpedance is based on the 
theory that the thorax is a blood filled cylinder. According to Ohm's law (resistance $=$ voltage/current) this model assumes that the impedance of thoracic tissue is parallel to that of blood. Blood related impedance changes repeat themselves with every heart beat and are linked to cardiac activity [7]. During systole, approximately 60 milliseconds after opening of the aortic valve, erythrocytes change their position from a random alignment to one parallel to the axial blood flow. This results in an increased conductivity allowing estimating the acceleration of flow through the aortic artery. However, early adoption of the technique showed diverging results with respect to precision and accuracy [8].

The basic equation was modified by Bernstein and Osypka, so that the maximum rate of change of impedance is related to the peak aortic blood acceleration [9]. The method used in the Aesculon was initially described as electrical cardiometry and it contributes the increase in conductivity to the orientation change of the red blood cells to determine the velocity of the blood flow, claiming to be a more accurate technique in a wide spectrum of patient conditions and patient populations including neonates and children [10, 11].

The bioimpedance method shows good results in clinical studies in young healthy volunteers. However, reliability in critically ill patients and in perioperative use is not proven and the available literature is inconclusive [12-15]. Moreover, until now it is unclear whether interruption of the skins integrity by a surgical incision could be a source of error in bioimpedance measurements. Recently Huang et al. found indications that skin incision can interfere with the bioimpedance technique [16].

Most available studies were performed in a neonatal and pediatric populations or during cardiac surgery, mainly in the postoperative period. Studies in adult patients undergoing high-risk cardiac surgery are lacking; therefore, we studied patient in the entire perioperative period $[10,11,17]$.

The aim of the present study was to compare the accuracy, precision, and trending ability of a thoracic bioimpedance technique with continuous pulmonary artery thermodilution before, during, and after surgical intervention.

Secondary aim was to assess whether interruption of the skins integrity and opening of the thoracic cavity by a surgical incision could be a source of error in bioimpedance measurements.

\section{Materials and Methods}

2.1. Study Design. The study protocol was approved by the institutional review board of the Maastricht University Medical Center+ (MEC 08-4-075) and written informed consent was obtained from each patient. In this prospective observational study, 50 adult patients planned for elective cardiac surgery were included. Exclusion criteria were age < 18 years and a contraindication for placement of a Swan Ganz catheter. As placement of a Swan Ganz catheter was needed to retrieve $\mathrm{CO}$ measurements, we only included patients receiving the $\mathrm{PAC}$ in accordance with routine care.

2.2. Measurement Protocol. After arrival in the operating theatre, patients were connected to standard monitoring including heart rate, invasive blood pressure, and oxygen saturation. Anesthesia was induced according to local protocols. Thereafter, after rubbing and cleaning the skin with alcohol to achieve a skin-to-electrode impedance as low as possible, four standard electrocardiogram electrodes were placed according to the manual of the Aesculon (Osypka Medical, Berlin, Germany) on the left part of the neck and on the left part of the thorax at the level of the processus xiphoideus. Then the Aesculon monitor was connected to the electrodes for continuous display of bioimpedance cardiac index $\left(\mathrm{CI}_{\mathrm{BIO}}\right)$. A PAC (Edwards Life Sciences Corporation, Irvine, CA, USA, continuous cardiac output VIP catheter with $\mathrm{SvO}_{2}$, model 746F8) was inserted via the right or left internal jugular vein in order to continuously measure thermodilution cardiac index $\left(\mathrm{CI}_{\mathrm{PAC}}\right)$. To exclude the possibility of incorrect measurements during rapid fluid injections and hemodynamic instability we measured $\mathrm{CI}_{\mathrm{BIO}}$ and $\mathrm{CI}_{\mathrm{PAC}}$ at hemodynamic stability, in the absence of engraving events (e.g., profound bleeding, hypotension, and arrhythmias) and during normothermia.

We used the mean of two measurements of continuous $\mathrm{CO}$. We used this as result per measurement and presented the results for each time point separately. There was no correction for repeated measurements applied because we analyzed the results per time point separately and assumed the measurements to be independent due to the extensive fluctuations.

Measurements were performed at 6 time points $(T)$ : after induction and prior to incision ( $T 1)$, prior to cannulation of the aorta (T2), 10 minutes after protamine administration (T3), 30 minutes after arrival in the ICU (T4), 1 hour after extubation (T5), and 1 day postoperatively at 08.00 a.m. (T6). In addition to $\mathrm{CI}$, heart rate, arterial blood pressure, and central and peripheral temperature were recorded at these time points.

2.3. Statistical Analysis. A sample size calculation was performed based on a previous study by Schmidt and coworkers [18]. Assuming a true difference between $\mathrm{CI}_{\mathrm{BIO}}$ and $\mathrm{CI}_{\mathrm{PAC}}$ of 0.5 liters $/ \mathrm{min} / \mathrm{m}^{2}$ and corresponding standard deviation (SD) of 1.0 liter $/ \mathrm{min} / \mathrm{m}^{2}$, a total number of 33 patients were needed to reject the null hypothesis that the difference is 0 with a power of 0.8 and type 1 error of 0.05 . To correct for loss to follow-up an additional 17 patients were included, resulting in 50 patients in total.

Data were checked for normality using the Shapiro-Wilk test histograms (visually), including the difference between $\mathrm{CI}_{\mathrm{BIO}}$ and $\mathrm{CI}_{\mathrm{PAC}}$. Descriptive analysis was performed using number $(\%)$ or mean \pm SD. Differences between the absolute CI measurements were assessed using the paired $t$-test. Accuracy and precision of $\mathrm{CI}_{\mathrm{BIO}}$ against $\mathrm{CI}_{\mathrm{PAC}}$ at the various time points were assessed using Bland-Altman analysis and plots showing the bias, limits of agreement (LOA), and percentage error $(\mathrm{PE})[19,20] . \mathrm{CI}_{\mathrm{BIO}}$ and $\mathrm{CI}_{\mathrm{PAC}}$ were considered interchangeable if the $\mathrm{PE}$ was $<30 \%$.

According to the literature, BMI might influence reliability of the bioimpedance measurements [18, 21]. Therefore additional analysis was performed comparing 16 patients with $\mathrm{BMI}>30$ with the remaining patients $(\mathrm{BMI} \leq 30)$. 
TABLE 1: Patient and surgery characteristics.

\begin{tabular}{lcc}
\hline & Mean $(\mathrm{sd})$ & Range \\
\hline Age (years) & $64.2(10.6)$ & 40 to 81 \\
Height $(\mathrm{m})$ & $1.72(0.1)$ & 1.55 to 1.94 \\
Weight $(\mathrm{kg})$ & $83.3(17.5)$ & 60 to 150 \\
$\mathrm{BMI}\left(\mathrm{kg} / \mathrm{m}^{2}\right)$ & $28.2(5.0)$ & 19.3 to 46.3 \\
& & $n$ \\
Sex m/f & & $35 / 15$ \\
$\mathrm{CABG}^{*}$ & & 31 \\
$\mathrm{AVR}^{\dagger}$ & & 10 \\
$\mathrm{MVR}^{\ddagger}$ & & 2 \\
CABG and AVR or MVR & 5 \\
Other & & 2 \\
\hline
\end{tabular}

In the formulas for the LOA and PE, a $t$-statistic of 2.02 was used at the various time points $(N=50)$ and 1.97 for pooled data $(N=300)$. To evaluate trending ability, fourquadrant plot and polar plot methodology was applied to the change in $\mathrm{CI}_{\mathrm{BIO}}$ and $\mathrm{CI}_{\mathrm{PAC}}$ between the time points $[22,23]$. Concerning polar plot analysis central zone data $(<10 \%$ change) were excluded because they introduce statistical noise. Angular bias is defined as the mean polar angle to the $0^{\circ}$ line. The radial LOA refer to the radial sector that contains 95\% of the data points. Polar concordance represents the percentage data points that lie within $\pm 30^{\circ}$. In case of good trending ability, most of the data points lie within this $30^{\circ}$ sector $[23,24]$. Trending ability of $\mathrm{CI}_{\mathrm{BIO}}$ was considered interchangeable with $\mathrm{CI}_{\mathrm{PAC}}$ if angular bias was between $-5^{\circ}$ and $+5^{\circ}$, with radial LOA between $-30^{\circ}$ and $+30^{\circ}$.

A $P$ value $<0.05$ was considered statistically significant and Bonferroni correction for multiple testing of the absolute differences at the six time points was applied $(P<0.05 / 6$ measurements $=0.008$ ).

Statistical analysis was carried out using SPSS software (SPSS Inc., Chicago, IL, USA) and Excel (Microsoft Corporation).

\section{Results}

Fifty patients undergoing cardiac surgery were included. The baseline characteristics are presented in Table 1 . The hemodynamic variables and temperatures are presented in Table 2. CI varied between 1.00 and $6.75\left(\mathrm{CI}_{\mathrm{PAC}}\right)$ and 0.93 and $7.25 \mathrm{~L} \mathrm{~min}^{-1}\left(\mathrm{CI}_{\mathrm{BIO}}\right)$. $\mathrm{CI}_{\mathrm{BIO}}$ and $\mathrm{CI}_{\mathrm{PAC}}$ were significantly different at each point except T6 (24 hours after surgery).

Differences were present at open chest (T2, T3) and closed chest $(T 5, T 6)$, respectively, indicating noninterchangeability between both techniques at both open and closed chest.

Bias between $\mathrm{CI}_{\mathrm{BIO}}$ and $\mathrm{CI}_{\mathrm{PAC}}$ was 0.52 liters $\min ^{-1} \mathrm{~m}^{-2}$, with LOA of $[-2.2 ; 1.1]$ liters $\mathrm{min}^{-1} \mathrm{~m}^{-2}$ (Table 3 ). Visual assessment of the Bland-Altman plots (Figure 1(a)) however shows that some agreement might be present at CI values between approximately 1.5 and $2.8 \mathrm{liters} / \mathrm{min} / \mathrm{m}^{2}$. At higher
$\mathrm{CI}$ values, the spread in the differences between $\mathrm{CI}_{\mathrm{BIO}}$ and $\mathrm{CI}_{\mathrm{PAC}}$ rapidly increases, especially at $T 1, T 3$, and $T 5$ (Figures $1(\mathrm{a})-1(\mathrm{~d}))$.

The percentage error between $\mathrm{CI}_{\mathrm{BIO}}$ and $\mathrm{CI}_{\mathrm{PAC}}$ was above the $30 \%$ agreement limit at every time point, including the lower limit of the $95 \%$ confidence intervals (Table 3 ).

The percentage error in patients with $\mathrm{BMI}>30$ ranged from $30 \%$ to $62 \%$ versus $52 \%$ to $79 \%$ in the patients with BMI $\leq 30$.

Trending ability was assessed in 89 pairs of changes in CI (Figure 2). Polar plot analysis in 77 data pairs outside the $10 \%$ exclusion zone showed an angular bias of $-12^{\circ}$. The radial LOA were $-55^{\circ}$ to $51^{\circ}$. All values were outside the boundaries for acceptable trending ability. Polar concordance at $30^{\circ}$ was $66 \%$. These results were outside the boundaries for acceptable trending ability. Four-quadrant plot analysis also showed poor trending ability. Data pairs outside the $15 \%$ exclusion zone showed a concordance of only $55 \%$ (Figure 3).

\section{Discussion}

The present study investigated the accuracy, precision, and trending ability of a thoracic impedance CO monitor (Aesculon) versus pulmonary artery thermodilution in patients undergoing cardiac surgery. Our results do not support interchangeability of both devices in this patient group during surgery as well as during the early postoperative period, as both PE and trending ability exceeded the clinically acceptable, predefined limits.

Visual interpretation of the Bland-Altman plots revealed only moderate agreement at CI between 1.5 and 2.8 liters $/ \mathrm{min} / \mathrm{m}^{2}$. However, dispersion increased with higher CI. This so-called "heteroscedasticity" or proportional spread indicates that significant imprecision arises with increasing $\mathrm{CO}$ and that the LOA may even be underestimated in the high $\mathrm{CO}$ range. Several studies compared $\mathrm{CO}$ derived from bioimpedance with intermittent or continuous pulmonary artery thermodilution. The results of agreement and precision were inconclusive, demonstrating a need for additional studies in this field before drawing definitive conclusion on the validity and reliability of the Aesculon device technique [17, 25-27]. Patients undergoing cardiac surgery still represent a clinically challenging patient population, with relevant perioperative morbidity and mortality.

In comparable high-risk surgical patients, the use of invasive hemodynamic monitoring in combination with goaldirected therapy has been shown to improve postoperative outcome $[28,29]$. In principle, thoracic bioimpedance represents a promising, noninvasive technique to be used in goaldirected strategies as the technique is noninvasive and independent from the observer and can be used also in patients at the ICU and medium care unit. Before implementation however, the reliability of the technology should be confirmed in the appropriate target patients. Apart from the clinical urgency, we decided to study patients undergoing cardiac surgery, because aortic cross clamping and clamp release induce profound changes in cardiac afterload followed by a profound ischemia-reperfusion injury. This implicates 
TABLE 2: Haemodynamic data.

\begin{tabular}{|c|c|c|c|c|c|c|}
\hline & $T 1$ & $T 2$ & T3 & $T 4$ & $T 5$ & T6 \\
\hline MAP & $71.7(8.8)$ & $70.2(10.0)$ & $70.9(9.6)$ & $75.8(12.0)$ & $74.7(7.5)$ & $79.6(11.4)$ \\
\hline HR & $61(11)$ & $68(17)$ & $80(11)$ & $80(12)$ & $90(14)$ & $86(12)$ \\
\hline CVP & $11(4.6)$ & $9(4.6)$ & $10(4.0)$ & $7(3.3)$ & $7(4.6)$ & $7(4.7)$ \\
\hline CI Aesculon & $1.8(0.5)$ & $1.9(0.7)$ & $2.2(0.8)$ & $2.2(0.7)$ & $2.8(1.1)$ & $2.6(0.7)$ \\
\hline CI Vigilance & $2.2(0.7)$ & $2.3(0.7)$ & $3.2(0.7)$ & $2.6(0.5)$ & $3.3(0.9)$ & $2.9(0.7)$ \\
\hline Temp central & $36.1(0.5)$ & $35.9(0.5)$ & $36.3(0.3)$ & $35.9(0.5)$ & $37.7(0.5)$ & $37.4(0.5)$ \\
\hline Temp peripheral & $30.9(1.8)$ & $32.1(1.6)$ & $33.3(2.2)$ & $32.9(1.7)$ & $34.7(1.8)$ & $34.3(1.9)$ \\
\hline
\end{tabular}

Mean (sd). MAP: mean arterial pressure ( $\mathrm{mmHg}$ ), HR: heart rate (beats $\left.\mathrm{min}^{-1}\right)$, CVP: central venous pressure ( $\mathrm{mmHg}$ ), CI: cardiac index (liters min ${ }^{-1} \mathrm{~m}^{-2}$ ), and Temp: central and peripheral body temperature $\left({ }^{\circ} \mathrm{C}\right)$. Time point $1(\mathrm{~T} 1)$ prior to surgery, after induction of anaesthesia; $T 2$ prior to cannulation of the aorta; T3 10 minutes after protamine administration; T4 30 minutes after arrival at the ICU; T5 one hour after extubation at the ICU; T6 first postoperative day, 8:00 a.m. in the ICU ward.

TABLE 3: Agreement results.

\begin{tabular}{|c|c|c|c|c|c|c|c|}
\hline & Pooled & $T 1$ & $T 2$ & $T 3$ & $T 4$ & T5 & T6 \\
\hline$N$ & 284 & 47 & 50 & 48 & 46 & 46 & 47 \\
\hline $\operatorname{Bias}\left(\mathrm{L} \mathrm{min}^{-1}\right)$ & -0.52 & -0.44 & -0.38 & -0.98 & -0.45 & -0.57 & -0.28 \\
\hline CI bias $\left(\mathrm{L} \mathrm{min}^{-1}\right)$ & -0.62 to -0.42 & -0.66 to -0.23 & -0.60 to -0.16 & -1.27 to -0.69 & -0.62 to -0.27 & -0.86 to -0.29 & -0.51 to -0.05 \\
\hline $\operatorname{LOA}\left(\mathrm{L} \mathrm{min}^{-1}\right)$ & -2.2 to 1.1 & -1.9 to 1.0 & -2.0 to 1.2 & -3.0 to 1.0 & -1.6 to 0.7 & -2.5 to 1.3 & -1.9 to 1.3 \\
\hline CI lower LOA $\left(\mathrm{L} \mathrm{min}^{-1}\right)$ & -2.3 to -2.0 & -2.3 to -1.5 & -2.4 to -1.6 & -3.5 to -2.5 & -1.9 to -1.3 & -3.0 to -2.0 & -2.3 to -1.5 \\
\hline CI upper LOA $\left(\mathrm{L} \mathrm{min}^{-1}\right)$ & 1.0 to 1.3 & 0.7 to 1.4 & 0.8 to 1.6 & 0.5 to 1.5 & 0.4 to 1.0 & 0.9 to 1.8 & 0.9 to 1.7 \\
\hline Percentage error (95\% CI) & $67(60$ to 73$)$ & 74 (56 to 92$)$ & 77 (58 to 96$)$ & 75 (56 to 93$)$ & 49 (37 to 62$)$ & $63(47$ to 79$)$ & $58(43$ to 72$)$ \\
\hline
\end{tabular}

Bias: difference between CI Aesculon and CI Vigilance. CI: $95 \%$ confidence interval. LOA: limits of agreement. Time point $(T) 1$ prior to surgery, after induction; T2 prior to cannulation of the aorta; T3 10 minutes after protamine administration; T4 30 minutes after arrival at the ICVU ward; T5 one hour after extubation at the ICU ward; T6 on day one postoperatively at 8:00 a.m. in the ICU ward.

the necessity of profound observation and monitoring of these patients.

With the emergence of devices for continuous CO measurement, research increasingly focuses at trending ability rather than accuracy of individual measurements at a specific time point [30]. Especially during and immediately after cardiac surgery, the CO change in time may be more interesting than its absolute value, since the patients usually suffer from cardiovascular comorbidity and limited cardiac reserve [27, 28]. Therefore, 4-quadrant plot and polar plot methodology was used to objectify trending ability of the new technique. The results indicate poor trending ability, which was expected regarding the results from Bland and Altman analysis. Usually, high mean errors indicate that there is no fixed deviation between the experimental and reference technique, which impedes tracking of changes in $\mathrm{CO}$ in a reliable manner.

One of the strengths of the present study is the fact that most studies comparing the Aesculon with an established technique focus on neonatal, critically ill, and postoperative patient populations $[10,11,15,25-27]$.

Furthermore, there is lack of reliable published data on the trending abilities of the Aesculon [22]. In the present study, a relatively homogeneous population of cardiac surgical patients was studied during the intra- and postoperative period. As we were interested in the ability of the thoracic impedance under different conditions, we analyzed baseline values before surgery (T1) compared to open chest $(T 2, T 3)$ and closed chest $(T 5, T 6)$ conditions. However, the results demonstrated that the noninterchangeability between both techniques was present during both, open and closed thorax. In addition, we obtained CI measurements before and after weaning from mechanical ventilation and extubation up to 24 hours after surgery to simulate a medium care environment. We found the difference between both techniques independent from these conditions above the accepted range of $30 \%$.

In our study population 16 patients had a BMI $>30$. The percentage error was slightly lower in the BMI $>30$ group but in both groups above the $30 \%$ limit. Clinical implication of these findings is questionable.

We included a substantial number of patients more than the calculated sample size to be sure we would have enough data. The concern was that we would have to deal with loss of data during observation at the ICU and possible failure of the Aesculon device. Accurate sampling at time points 5 and 6 also could be an expected problem.

Continuous pulmonary artery thermodilution was used as the reference technique. We used the mean of two measurements of continuous $\mathrm{CO}$. Although thermodilution $\mathrm{CO}$ (TDCO) is considered as best acceptable clinical standard method for CO measurement, TDCO has an intrinsic variability, in particular during changing hemodynamic conditions and high CO [31]. Compared to the single bolus method, the continuous $\mathrm{CO}$ method has limited accuracy particularly during hemodynamic instability and hypothermia [31-33]. 


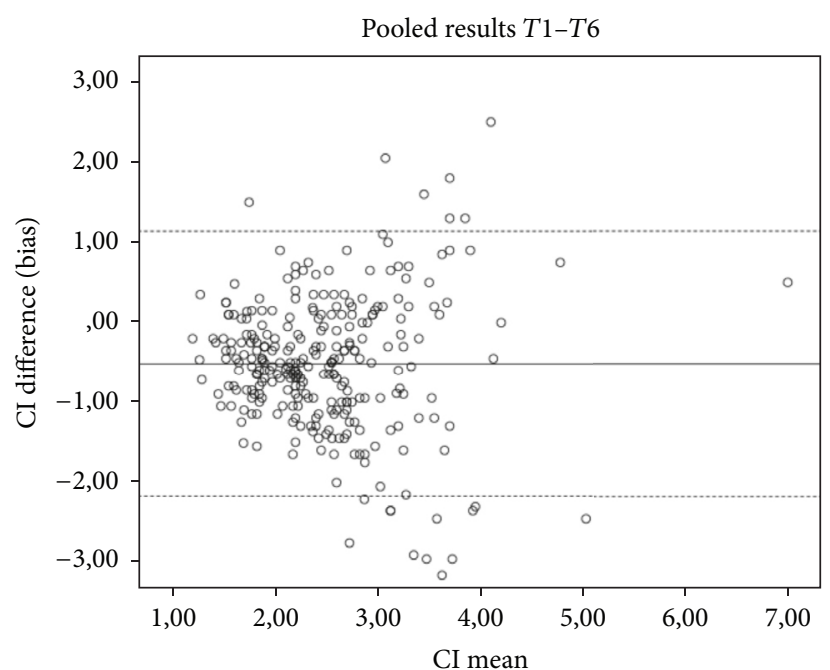

(a) Pooled results

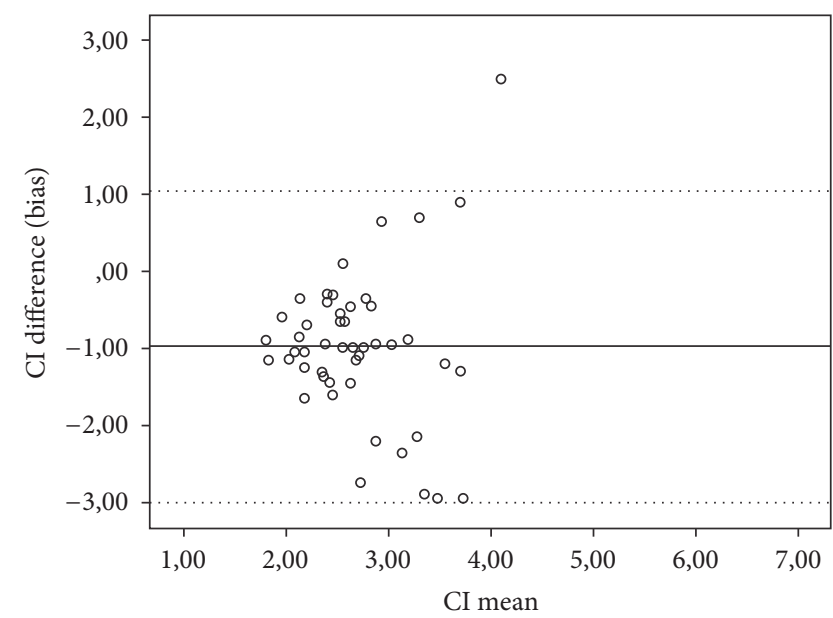

(c) Time point 3

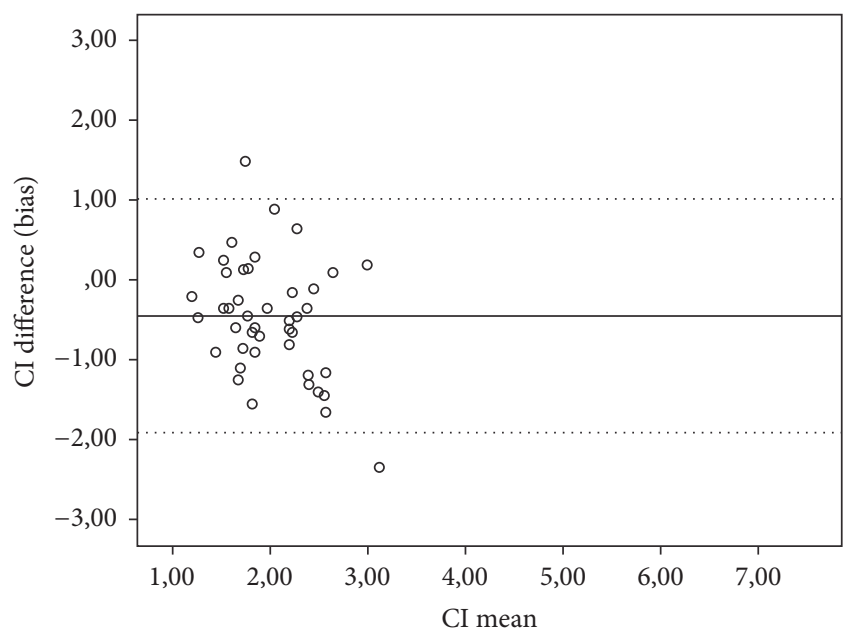

(b) Time point 1

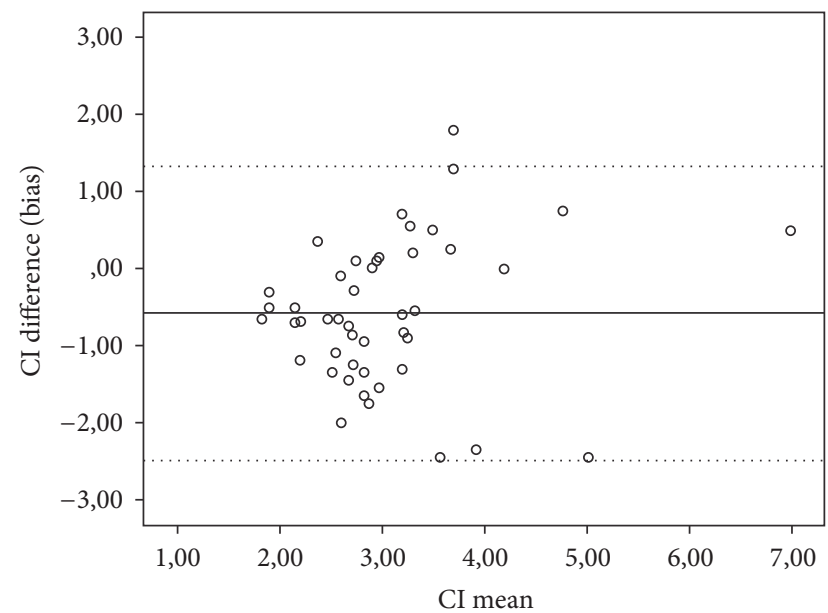

(d) Time point 5

FIGURE 1: Bland and Altman plot. Fixed line indicates mean difference, and dotted lines indicate limits of agreement. (a) Pooled results, time point $(T) 1$ prior to surgery, after induction; T3 10 minutes after protamine administration; T5 one hour after extubation at the ICU ward.

Being a combined measure of precision, the mean error may also be the result of variability of the reference technique $[29,34]$.

The pulmonary artery thermodilution catheter (PAC) has a precision of $\pm 20 \%$ or less. The combination of two precisions of $\pm 20 \%$ equates to a total error of $\pm 28.3 \%$, which is commonly rounded up to $\pm 30 \%$ and is clinically acceptable $[35,36]$.

Moreover, the generally accepted range for bias (20\%) and mean error $(30 \%)$ are still a matter of discussion. The relatively large limit for bias was chosen to take possible hemodynamic changes into account. Depending on the clinical context, the limits can be defined more narrowly or even less narrowly. In general, it is advisable to use predefined criteria for acceptable bias and LOA in each method comparison study, since Bland and Altman analysis does not provide definitive answers. The same applies to the boundaries for trend parameters.
Our secondary aim was to assess whether the surgical incision, and therefore the interruption of the continuity of the skin of the thoracic cavity and opening of the cavity itself, could be an important factor in the reported discrepancy between the two instruments. Also, our study was not specifically designed to answer this question; we could not find any evidence for this hypothesis. The percentage error was always above $30 \%$, being even above $70 \%$ in $T 1$ and $T 3$. Also the bias was very unpredictable, and the highest and the almost lowest value were measured during incision of the skin.

\section{Conclusion}

Analysis according to Bland-Altman shows that the Aesculon cannot be used interchangeably with the PAC in the operation theatre as a tool for beat to beat clinical decision making. We found no effect of skin interruption on accuracy, precision, and trending ability of the investigated technique. 


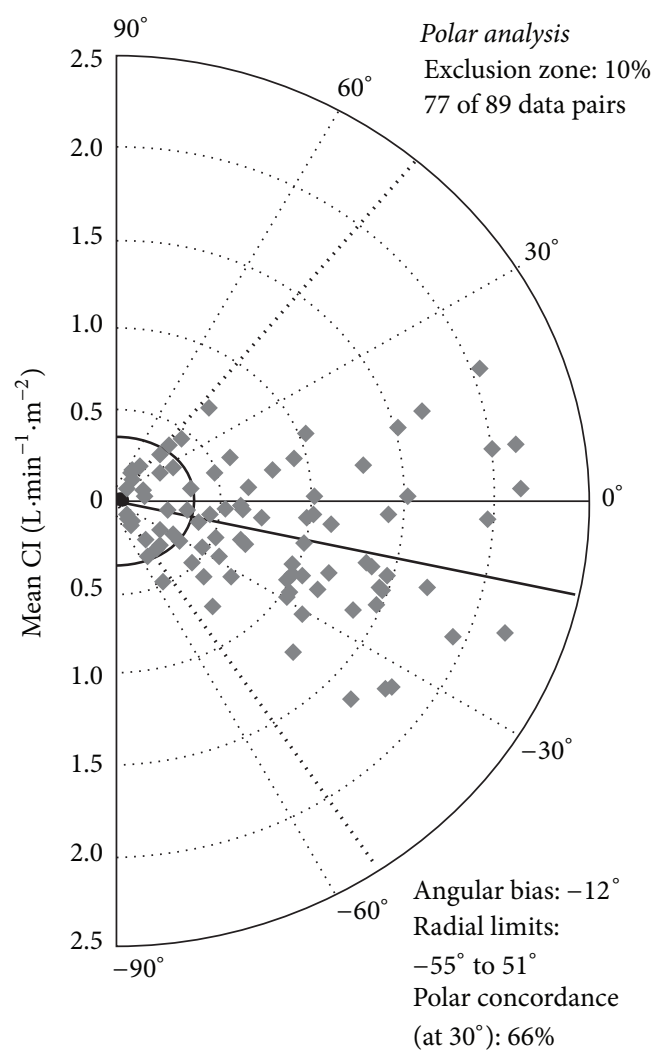

Figure 2: Polar plot analysis. In the polar plot, the distance from the centre represents the magnitude of the change in CI, whereas the angle with $0^{\circ}$ line refers to its direction. Increases and decreases in CI are shown together in the so-called "half-moon" design. The mean change in $\mathrm{CI}$ (mean $\Delta \mathrm{CI}$ ) was used, with a $10 \%$ exclusion zone. Ideally, the mean angle of all data points is $0^{\circ}$, with at least $95 \%$ of all data points within the $-30^{\circ}$ to $30^{\circ}$ sector (small dotted lines). Angular bias and radial LOA are depicted (solid line and thick dotted lines).

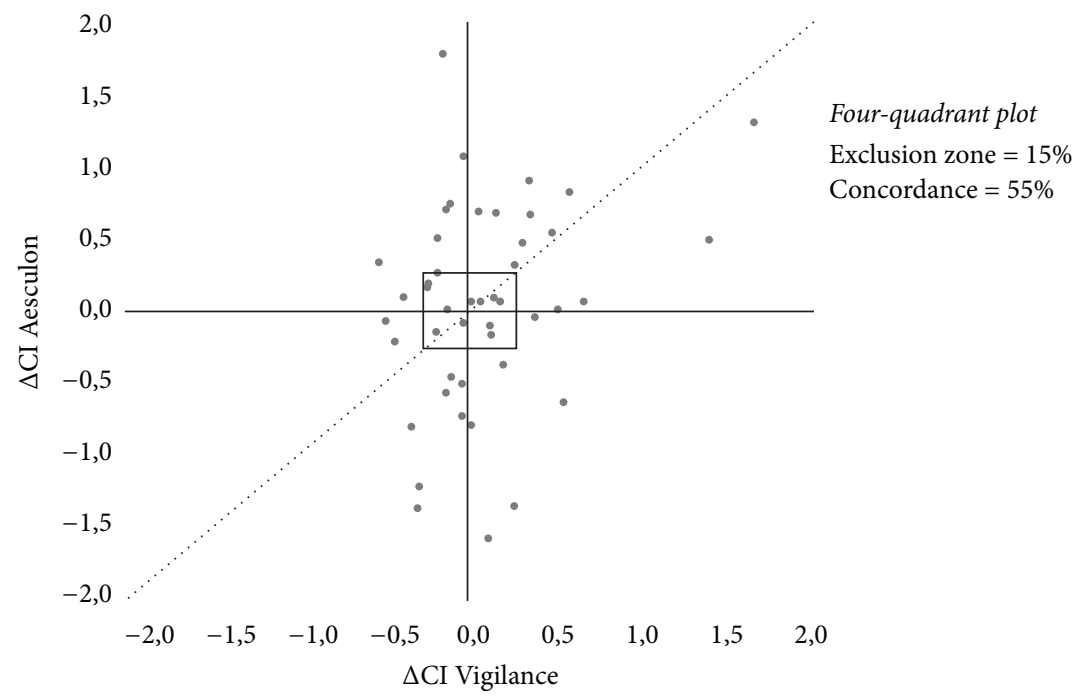

FIGURE 3: Four-quadrant plot analysis. Assessment of trending ability for pooled data. In the four-quadrant plot, the change in CI Vigilance ${ }^{\mathrm{TM}}$ ( $x$-axis) is plotted against the change in CI Aesculon ( $y$-axis). Ideally, all data points lie along the line of identity " $y$ 1/4 $x$ " (dotted line). The central square refers to the $15 \%$ exclusion zone. The zero axes " $y 1 / 40$ " and " $x 1 / 40$ " cross in the centre of the plot, creating four quadrants. In the right upper and left lower quadrants, CI Aesculon and CI Vigilance agree, which means that CI Aesculon and CI Vigilance change in the same direction. The concordance refers to the percentage of data points in these quadrants. 


\section{Abbreviations}

PAC: Pulmonary artery catheter

EC: Electrical cardiometry

CO: Cardiac output

CI: Cardiac index

ICU: Intensive care unit

T: $\quad$ Time point

SPSS: Statistical package for social science

SD: Standard deviation

B-A: Bland and Altman.

\section{Additional Points}

Key Messages. The bioimpedance cannot be used interchangeably with the PAC during cardiac surgery.

\section{Conflicts of Interest}

The authors declare that they have no conflicts of interest.

\section{Authors' Contributions}

P. B. W. Cox contributed to the design of the study, carried out the analyses, interpreted the data, and wrote the manuscript. M. Theunissen interpreted a part of the data and reviewed the manuscript. M. D. Lancé participated in its design and coordination. W. F. F. A. Buhre reviewed the manuscript critically. A. M. den Ouden helped in analyzing the data and contributed to the draft and improvement of the manuscript. M. A. E. Marcus contributed in initial design and reviewed the study. A. G. H. Kessels contributed in statistical analysis and data interpretation. L. J. Montenij advised substantially on statistical analysis and data interpretation.

\section{References}

[1] A. F. Connors Jr., T. Speroff, N. V. Dawson et al., "The effectiveness of right heart catheterization in the initial care of critically ill patients," Journal of the American Medical Association, vol. 276, no. 11, pp. 889-897, 1996.

[2] E. C. Rackow, "Pulmonary Artery Catheter Consensus Conference," Critical Care Medicine, vol. 25, no. 6, p. 901, 1997.

[3] B. Afessa, S. Spencer, W. Khan, M. LaGatta, L. Bridges, and A. $\mathrm{X}$. Freire, "Association of pulmonary artery catheter use with in-hospital mortality," Critical Care Medicine, vol. 29, no. 6, pp. 1145-1148, 2001.

[4] H. J. Swan, "The pulmonary artery catheter in anesthesia practice. 1970.," Anesthesiology., vol. 103, no. 4, pp. 890-893, 2005.

[5] S. S. Rajaram, N. K. Desai, A. Kalra et al., "Pulmonary artery catheters for adult patients in intensive care.," Cochrane database of systematic reviews (Online), vol. 2, p. CD003408, 2013.

[6] E. E. C. De Waal, F. Wappler, and W. F. Buhre, "Cardiac output monitoring," Current Opinion in Anaesthesiology, vol. 22, no. 1, pp. 71-77, 2009.

[7] H. H. Woltjer, H. J. Bogaard, and P. M. J. M. De Vries, "The technique of impedance cardiography," European Heart Journal, vol. 18, no. 9, pp. 1396-1403, 1997.
[8] E. E. C. De Waal, M. K. Konings, C. J. Kalkman, and W. F. Buhre, "Assessment of stroke volume index with three different bioimpedance algorithms: Lack of agreement compared to thermodilution," Intensive Care Medicine, vol. 34, no. 4, pp. 735739, 2008.

[9] D. O. Bernstein, "Apparatus and method for determining an approximation of the stroke volume and the cardiac output of the heart," US patent 6,511,438 B2, 2003.

[10] R. Rauch, E. Welisch, N. Lansdell et al., "Non-invasive measurement of cardiac output in obese children and adolescents: Comparison of electrical cardiometry and transthoracic Doppler echocardiography," Journal of Clinical Monitoring and Computing, vol. 27, no. 2, pp. 187-193, 2013.

[11] J. Wong, M. S. D. Agus, and G. M. Steil, "Cardiac parameters in children recovered from acute illness as measured by electrical cardiometry and comparisons to the literature," Journal of Clinical Monitoring and Computing, vol. 27, no. 1, pp. 81-91, 2013.

[12] A. C. Perrino Jr., A. Lippman, C. Ariyan, T. Z. O'Connor, and M. Luther, "Intraoperative cardiac output monitoring: Comparison of impedance cardiography and thermodilution," Journal of Cardiothoracic and Vascular Anesthesia, vol. 8, no. 1, pp. 24-29, 1994.

[13] E. Barin, D. G. Haryadi, S. I. Schookin et al., "Evaluation of a thoracic bioimpedance cardiac output monitor during cardiac catheterization," Critical Care Medicine, vol. 28, no. 3, pp. 698$702,2000$.

[14] M. Imhoff, J. H. Lehner, and D. Lohlein, "Noninvasive wholebody electrical bioimpedance cardiac output and invasive thermodilution cardiac output in high-risk surgical patients," Critical Care Medicine, vol. 28, no. 8, pp. 2812-2818, 2000.

[15] N. Zoremba, J. Bickenbach, B. Krauss, R. Rossaint, R. Kuhlen, and G. Schälte, "Comparison of electrical velocimetry and thermodilution techniques for the measurement of cardiac output," Acta Anaesthesiologica Scandinavica, vol. 51, no. 10, pp. 1314-1319, 2007.

[16] L. Huang, L. A. H. Critchley, and J. Zhang, "Major upper abdominal surgery alters the calibration of BioReactance cardiac output readings, the NICOM, when comparisons are made against suprasternal and esophageal Doppler Intraoperatively," Anesthesia and Analgesia, vol. 121, no. 4, pp. 936-945, 2015.

[17] A. Faini, S. Omboni, M. Tifrea, S. Bubenek, O. Lazar, and G. Parati, "Cardiac index assessment: Validation of a new noninvasive very low current thoracic bioimpedance device by thermodilution," Blood Pressure, vol. 23, no. 2, pp. 102-108, 2014.

[18] B. J. M. Van Der Meer, J. P. P. M. De Vries, W. O. Schreuder, E. R. Bulder, L. Eysman, and P. M. J. M. De Vries, "Impedance cardiography in cardiac surgery patients: Abnormal body weight gives unreliable cardiac output measurements," Acta Anaesthesiologica Scandinavica, vol. 41, no. 6, pp. 708-712, 1997.

[19] J. M. Bland and D. G. Altman, "Statistical methods for assessing agreement between two methods of clinical measurement," The Lancet, vol. 1, no. 8476, pp. 307-310, 1986.

[20] J. M. Bland and D. G. Altman, "Agreed statistics: measurement method comparison," Anesthesiology, vol. 116, no. 1, pp. 182-185, 2012.

[21] B. J. M. Van Der Meer, H. H. Woltjer, A. M. Sousman et al., "Impedance cardiography. Importance of the equation and the electrode configuration," Intensive Care Medicine, vol. 22, no. 10, pp. 1120-1124, 1996.

[22] L. A. Critchley, A. Lee, and A. M.-H. Ho, "A critical review of the ability of continuous cardiac output monitors to measure trends 
in cardiac output," Anesthesia and Analgesia, vol. 111, no. 5, pp. 1180-1192, 2010.

[23] L. A. Critchley, X. X. Yang, and A. Lee, "Assessment of trending ability of cardiac output monitors by polar plot methodology," Journal of Cardiothoracic and Vascular Anesthesia, vol. 25, no. 3, pp. 536-546, 2011.

[24] L. J. Montenij, W. F. Buhre, S. A. De Jong et al., "Arterial pressure waveform analysis versus thermodilution cardiac output measurement during open abdominal aortic aneurysm repair: A prospective observational study," European Journal of Anaesthesiology, vol. 32, no. 1, pp. 13-19, 2015.

[25] D. Mekiš, M. Kamenik, V. Starc, and S. Jeretin, "Cardiac output measurements with electrical velocimetry in patients undergoing CABG surgery: A comparison with intermittent thermodilution," European Journal of Anaesthesiology, vol. 25, no. 3, pp. 237-242, 2008.

[26] B. D. Spiess, M. A. Patel, L. O. Soltow, and I. H. Wright, "Comparison of bioimpedance versus thermodilution cardiac output during cardiac surgery: Evaluation of a second-generation bioimpedance device," Journal of Cardiothoracic and Vascular Anesthesia, vol. 15, no. 5, pp. 567-573, 2001.

[27] M. Heringlake, U. Handke, T. Hanke et al., "Lack of agreement between thermodilution and electrical velocimetry cardiac output measurements," Intensive Care Medicine, vol. 33, no. 12, pp. 2168-2172, 2007.

[28] R. Pearse, D. Dawson, J. Fawcett, A. Rhodes, R. M. Grounds, and E. D. Bennett, "Early goal-directed therapy after major surgery reduces complications and duration of hospital stay. A randomised, controlled trial [ISRCTN38797445].," Critical care (London, England), vol. 9, no. 6, pp. R687-693, 2005.

[29] A. Rhodes, M. Cecconi, M. Hamilton et al., "Goal-directed therapy in high-risk surgical patients: A 15-year follow-up study," Intensive Care Medicine, vol. 36, no. 8, pp. 1327-1332, 2010.

[30] S. W. M. C. Maass, P. M. H. J. Roekaerts, and M. D. Lancé, "Cardiac output measurement by bioimpedance and noninvasive pulse contour analysis compared with the continuous pulmonary artery thermodilution technique," Journal of Cardiothoracic and Vascular Anesthesia, vol. 28, no. 3, pp. 534-539, 2014.

[31] L. Jacquet, G. Hanique, D. Glorieux, P. Matte, and M. Goenen, "Analysis of the accuracy of continuous thermodilution cardiac output measurement. Comparison with intermittent thermodilution and Fick cardiac output measurement," Intensive Care Medicine, vol. 22, no. 10, pp. 1125-1129, 1996.

[32] B. W. Böttiger, H. Rauch, H. Böhrer et al., "Continuous versus intermittent cardiac output measurement in cardiac surgical patients undergoing hypothermic cardiopulmonary bypass," Journal of Cardiothoracic and Vascular Anesthesia, vol. 9, no. 4, pp. 405-411, 1995.

[33] K. Ameloot, I. Meex, C. Genbrugge et al., "Accuracy of continuous thermodilution cardiac output monitoring by pulmonary artery catheter during therapeutic hypothermia in post-cardiac arrest patients," Resuscitation, vol. 85, no. 9, pp. 1263-1268, 2014.

[34] J. T. Ho, M. J. Chapman, S. O'Connor et al., "Characteristics of plasma NOx levels in severe sepsis: High interindividual variability and correlation with illness severity, but lack of correlation with cortisol levels," Clinical Endocrinology, vol. 73, no. 3, pp. 413-420, 2010.

[35] M. Cecconi, A. Rhodes, J. Poloniecki, G. Della Rocca, and R. M. Grounds, "Bench-to-bedside review: the importance of the precision of the reference technique in method comparison studies-with specific reference to the measurement of cardiac output.," Critical care (London, England), vol. 13, no. 1, p. 201, 2009.

[36] L. A. H. Critchley and J. A. J. H. Critchley, "A meta-analysis of studies using bias and precision statistics to compare cardiac output measurement techniques," Journal of Clinical Monitoring and Computing, vol. 15, no. 2, pp. 85-91, 1999. 


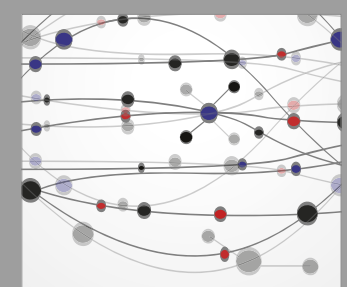

The Scientific World Journal
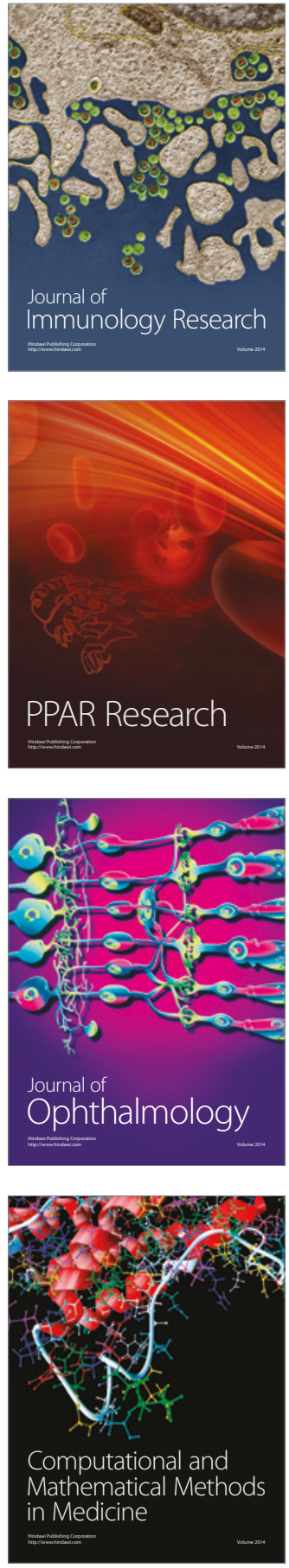

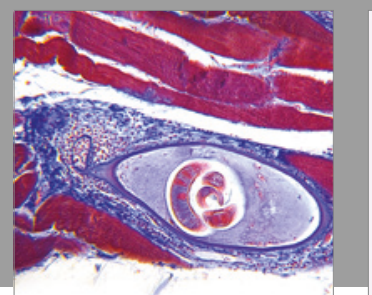

Gastroenterology Research and Practice
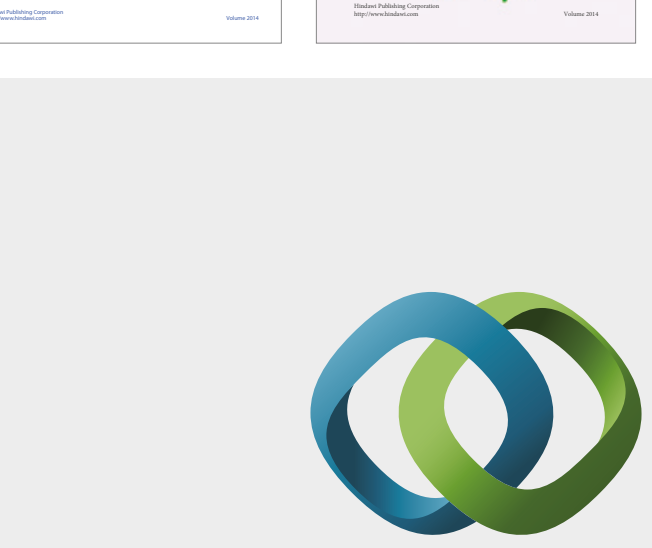

\section{Hindawi}

Submit your manuscripts at

https://www.hindawi.com
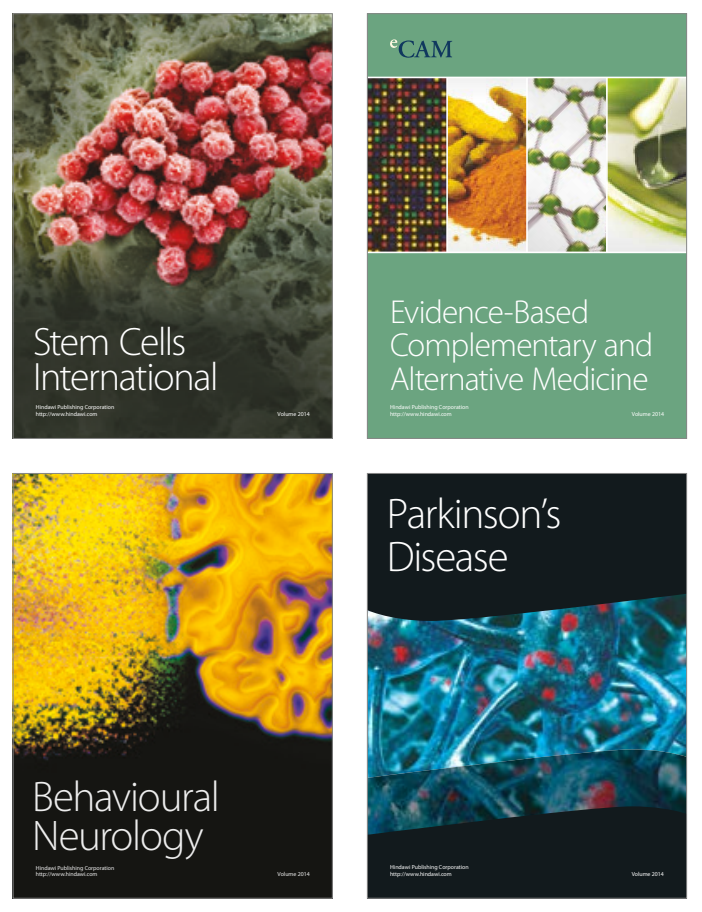
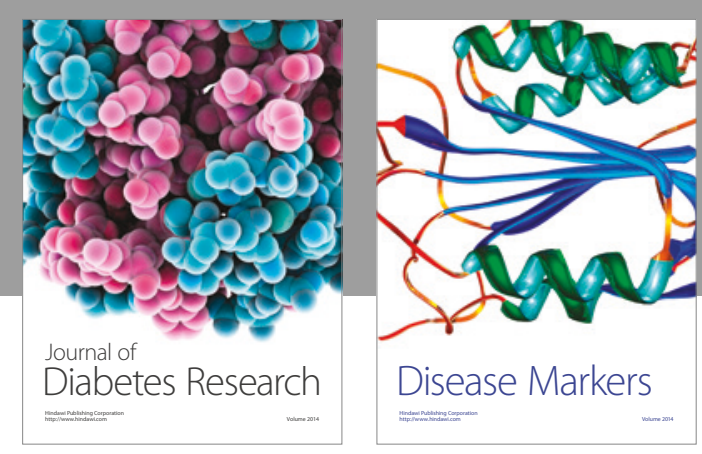

Disease Markers
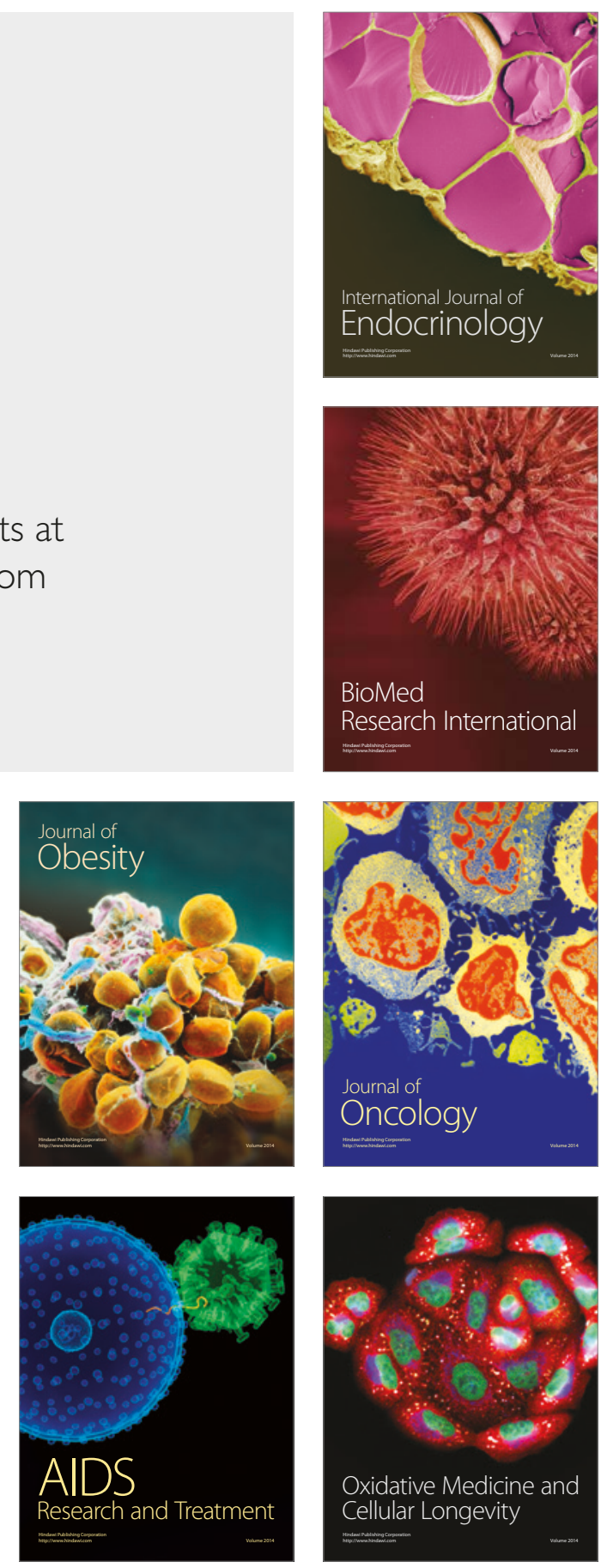